\title{
OPTIMIZATION OF STEADY-STATE FLOW OF INCOMPRESSIBLE FLUIDS *
}

\author{
Tomáš Roubíček \\ Mathematical Institute, Charles University, Sokolovská 83, CZ-186 75 Praha 8, and \\ Institute of Information Theory and Automation, Academy of Sciences, Pod vodárenskou \\ věži 4, CZ-182 08 Praha 8, Czech Republic. \\ tomas.roubicek@mff.cuni.cz
}

\begin{abstract}
An optimal boundary-control problem for the steady-state buoyancydriven flow of an incompressible, heat conductive fluid is investigated as far as optimality conditions and an increment formula concerns. The controlled system covers, in particular, the Oberbeck-Boussinesq model. Regularity of the Navier-Stokes system coupled with heat equation and its adjoint system is substantially used.
\end{abstract}

Keywords: Buoyancy-driven flow, optimality conditions, increment formula.

\section{Introduction}

Optimal control problem of steady-state incompressible flow coupled with heat equation was already studied in $[2,4,8,9,19,20]$. The isothermal case (i.e. without heat equation) was studied, e.g., in $[1,3,5,10,15,16,17,27]$ and [22; Section III.11]. Due to generality of the controlled system (1) below, this contribution covers a lot of these results as a special case.

Besides, optimal control of evolutionary Navier-Stokes system was treated in a lot of other works, see e.g. monographs $[11,12,22]$ and references therein.

\section{The controlled system}

Our a bit academical problem can be interpreted, after necessary simplifications, as a flow of, say, water heated up from outside to prepare a

*This research was partly covered by the grants 201/00/0768 (GA ČR), A 1075005 (GA AV ČR), and MSM 11320007 (MŠMT ČR).

The original version of this chapter was revised: The copyright line was incorrect. This has been corrected. The Erratum to this chapter is available at DOI: 10.1007/978-0-387-35690-7_44 
morning tee in a completely closed container $\Omega$. Anyhow, one can think, e.g., about transport in a high-pressure vapor reactor $[19,20]$ or flow a melted steel in a mold during a casting process, too.

We consider $\Omega$ a bounded smooth domain in $\mathbb{R}^{d}, d \leq 3$. To cover various possibilities, we consider the following fairly general system:

$$
\begin{aligned}
(u \cdot \nabla) u-\nu \Delta u+\nabla p & =g\left(1-\alpha_{0} \theta\right), \\
\operatorname{div} u & =0 \\
u \cdot \nabla \theta-\kappa \Delta \theta & =\alpha_{1}|\nabla u|^{2}+\alpha_{2} \theta g \cdot u,
\end{aligned}
$$

where $u$ is the velocity vector field, $p$ the pressure, $\theta$ temperature, and $g$ an external (e.g. gravity) force. The material parameters are: $\nu$ the viscosity, $\kappa$ heat conductivity, $\alpha_{0}$ linearized relative mass density variation with respect to temperature, $\alpha_{1}$ reflects dissipation effects, while $\alpha_{2}$ expresses adiabatic heat effects. For a rigorous derivation of a system like (1) we refer to Kagei, Růžička and Thäter [21; System (16)] or Rajagopal, Růžička and Srinivasa [25]: it is shown that the coefficient $\alpha_{1}$ depends on Ostrach's dissipation number, while the coefficient $\alpha_{2}$ depends also on the Reynolds and the Prandtl numbers. The conventional Oberbeck-Boussinesq model uses $\alpha_{1}=\alpha_{2}=0$. For derivation of the model with $\alpha_{1}>0$ but $\alpha_{2}=0$ see [24; Chap.I].

The system should be completed by boundary conditions. For simplicity, we will consider no-slip boundary condition for velocity and Newton's boundary condition for temperature, i.e.

$$
u=0, \quad \kappa \frac{\partial \theta}{\partial n}+b \theta=h \quad \text { on } \Gamma,
$$

with $n$ being the unit outward normal to the boundary $\Gamma:=\partial \Omega$ of $\Omega$.

We call the pair $(u, \theta) \in W_{0, \text { DIV }}^{1,2}\left(\Omega ; \mathbb{R}^{d}\right) \times W^{1,2}(\Omega)$ a weak solution to the boundary-value problem (1)-(2) if

$$
\begin{aligned}
& ((u \cdot \nabla) u, v)+\nu(\nabla u, \nabla v)+\kappa(\nabla \theta, \nabla \tilde{v})+(u \cdot \nabla \theta, \tilde{v}) \\
& +(b \theta, \tilde{v})_{\Gamma}-\left(\left(1-\alpha_{0} \theta\right) g, v\right)-\left(\alpha_{1}|\nabla u|^{2}+\alpha_{2} \theta g \cdot u, \tilde{v}\right) \\
& -(h, \tilde{v})_{\Gamma}=0 \quad \forall v \in W_{0, \mathrm{DIV}}^{1,2}\left(\Omega ; \mathbb{R}^{d}\right), \tilde{v} \in W^{1,2}(\Omega),
\end{aligned}
$$

where $W_{0, \mathrm{DIV}}^{1,2}\left(\Omega ; \mathbb{R}^{d}\right):=\left\{v \in W_{0}^{1,2}\left(\Omega ; \mathbb{R}^{d}\right) ; \operatorname{div} v=0\right\},(\cdot, \cdot)$ denotes the scalar product in $L^{2}(\Omega)$ or $L^{2}\left(\Omega ; \mathbb{R}^{d}\right)$ or $L^{2}\left(\Omega ; \mathbb{R}^{d \times d}\right)$ and $(\cdot, \cdot)_{\Gamma}$ is the scalar product in $L^{2}(\Gamma)$. Moreover, in what follows we will denote by $\|\cdot\|_{p}$ the norm of $L^{p}(\Omega)$ or $L^{p}\left(\Omega ; \mathbb{R}^{d}\right)$ or $L^{p}\left(\Omega ; \mathbb{R}^{d \times d}\right)$ and by $\|\cdot\|_{p, \Gamma}$ the norm of $L^{p}(\Gamma)$. Besides, $N_{\Gamma}$ will be the norm of the trace operator $W^{1,2}(\Omega) \rightarrow L^{2}(\Gamma)$, and $N_{p}$ and $N_{p, q}$ will denote the norm of the embedding $W_{0}^{1,2}(\Omega) \subset L^{p}(\Omega)$ and $L^{p}(\Omega) \subset L^{q}(\Omega)$, respectively. 
The existence of a weak solution to the whole problem (1)-(2) is not automatic unless $\alpha_{1}=\alpha_{2}=0$. Its uniqueness is even more delicate because, due to the quadratic terms on the right-hand side of (1c), we lack any global a-priori estimate if $\alpha_{1} \neq 0 \neq \alpha_{2}$ and therefore we can get the uniqueness only of those solutions whose energy does not exceed certain limits.

For some $h_{\min }<h_{\max }$, we denote the set of admissible $h$ 's in (2) by

$$
\mathfrak{H}_{\text {ad }}=\left\{h \in L^{\infty}(\Gamma) ; h_{\min } \leq h(x) \leq h_{\max } \text { for a.a. } x \in \Gamma\right\} .
$$

Proposition 1 Let $\varrho>N_{2} N_{\Gamma} c_{\mathrm{P}}^{-1} \max \left(\left|h_{\min }\right|,\left|h_{\max }\right|\right) \sqrt{\operatorname{meas}_{\mathrm{d}-1}(\Gamma)}$ and $\alpha_{0}, h_{\min }, h_{\text {max }} \in \mathbb{R}$ be arbitrary, where $c_{\mathrm{P}}=c_{\mathrm{P}}\left(\kappa, b_{\min }, \Omega\right)$ is the constant from the Poincaré inequality $c_{\mathrm{P}}\|\theta\|_{W^{1,2}(\Omega)}^{2} \leq \kappa\|\nabla \theta\|_{2}^{2}+b_{\min }\|\theta\|_{2, \Gamma}^{2}$. Let the assumptions

$$
\begin{aligned}
& \alpha_{0} N_{2} N_{4}^{2}\|g\|_{\infty} \varrho<\nu, \\
& g \in L^{\infty}\left(\Omega ; \mathbb{R}^{d}\right) \text { has a potential, i.e. } g=\nabla \varphi, \\
& \alpha_{1} \geq 0, \alpha_{2} \geq 0 \text { sufficiently small, } \\
& b_{\max } \geq b(x) \geq b_{\min }>0 \text { for a.a. } x \in \Gamma
\end{aligned}
$$

be satisfied. Then, for each $h \in \mathfrak{H}_{\text {ad }}$, there exists a weak solution $(u, \theta)$ to (1)-(2) satisfying $\|\theta\|_{2} \leq \varrho$. Moreover, if

$$
\nu, \kappa, b_{\min } \text { are sufficiently large, }
$$

this solution is determined uniquely and the mapping $h \mapsto(u, \theta): L^{2}(\Omega) \rightarrow$ $W^{1,2}\left(\Omega ; \mathbb{R}^{d+1}\right)$, restricted on $\mathfrak{H}_{\mathrm{ad}}$, is Lipschitz continuous and (weak,norm)continuous.

Proof. For the existence, we use a Schauder fixed-point argument similarly (but not entirely the same) as in [26]: we take $\vartheta \in L^{2}(\Omega),\|\vartheta\|_{2} \leq \varrho$, find a unique $u \in W_{0, \mathrm{DIV}}^{1,2}\left(\Omega ; \mathbb{R}^{d}\right)$ solving (in the weak sense) the NavierStokes system

$$
(u \cdot \nabla) u-\nu \Delta u+\nabla p=g\left(1-\alpha_{0} \vartheta\right), \quad \operatorname{div} u=0,\left.\quad u\right|_{\Gamma}=0,
$$

which is possible thanks to the assumption (5a). Testing (3) by $(v, \tilde{v}):=$ $(u, 0)$ (note that the term $(g, v)=(\nabla \varphi, v)=-(\varphi, \operatorname{div} v)=0$ in $(3)$ vanishes due to $(5 b))$, the basic a-priori estimate

$$
\|\nabla u\|_{2} \leq \frac{N_{2}}{\nu}\|g\|_{\infty} \alpha_{0} \varrho=: C_{1}
$$


is easily obtained. Then we take the unique $\theta \in W^{1,2}(\Omega)$ solving (in the weak sense) the heat equation

$$
\begin{aligned}
& u \cdot \nabla \theta-\kappa \Delta \theta=f, \quad \kappa \frac{\partial \theta}{\partial n}+\left.b \theta\right|_{\Gamma}=h, \\
& \text { with } f \equiv f(u, \vartheta):=\alpha_{1}|\nabla u|^{2}+\alpha_{2} \vartheta g \cdot u,
\end{aligned}
$$

The (nowadays standard) regularity result

$$
\|u\|_{\infty} \leq c\|u\|_{W^{2,2}\left(\Omega ; \mathbb{R}^{d}\right)} \leq C_{2} \equiv C_{2}(\Omega, \varrho)
$$

is known, see e.g. [7] or [14; Chap.VIII, Thm.5.2]. This shows, in particular, that $f \in L^{2}(\Omega)$ so that the unique weak solution to (9) certainly does exist. This regularity together with the compact embedding $W^{2,2}\left(\Omega ; \mathbb{R}^{d}\right) \subset W^{1,6-\varepsilon}\left(\Omega ; \mathbb{R}^{d}\right)$ shows that the mapping $\vartheta \mapsto f(u(\vartheta), \vartheta)$ : $L^{2}(\Omega) \rightarrow L^{2}(\Omega)$ is (weak,weak)-continuous; the limit passage in the term $\alpha_{2} \vartheta g \cdot u$ is easy because, due to (10) and compactness of the embedding $W^{2,2}(\Omega) \subset L^{\infty}(\Omega)$, the mapping $\vartheta \mapsto u: L^{2}(\Omega) \rightarrow L^{\infty}\left(\Omega ; \mathbb{R}^{d}\right)$ is (weak,norm)-continuous. By standard arguments, $f \mapsto \theta: L^{2}(\Omega) \rightarrow$ $W^{1,2}(\Omega)$ is (weak,norm)-continuous. Altogether, $\vartheta \mapsto \theta: L^{2}(\Omega) \rightarrow$ $L^{2}(\Omega)$ is (weak,norm)-continuous. Note that, if $\alpha_{1}=\alpha_{2}=0$, then

$$
c_{\mathrm{P}}\|\theta\|_{2} \leq N_{2} N_{\Gamma} \max \left(\left|h_{\min }\right|,\left|h_{\max }\right|\right) \sqrt{\operatorname{meas}_{d-1}(\Gamma)} .
$$

If $\varrho$ is taken as assumed and (5c) holds, the mapping $\vartheta \mapsto \theta$ maps the ball $\left\{\vartheta \in L^{2}(\Omega) ;\|\vartheta\|_{2} \leq \varrho\right\}$ into itself and, by Schauder's theorem, it has a fixed point $\theta$. Then $\theta=\vartheta$ together with the corresponding $u=u(\vartheta)$ from (7) solves (1)-(2).

Now, we will show the claimed Lipschitz-continuous dependence on $h$ of this fixed point and, as a by-product, we get its uniqueness as well as its (weak,norm)-continuity. Write the integral identity (3) for two right-hand sides, say $h_{1}$ and $h_{2}$. Then $\left(u_{1}, \theta_{1}\right)$ and $\left(u_{2}, \theta_{2}\right)$ will denote (some of) the corresponding solutions. Now we subtract these identities (3) and test it by $v:=u_{1}-u_{2}$ and $\tilde{v}=\theta_{1}-\theta_{2}$. Then, abbreviating shortly $u_{12} \equiv u_{1}-u_{2}, \theta_{12} \equiv \theta_{1}-\theta_{2}$, and $h_{12} \equiv h_{1}-h_{2}$ and using the identities

$$
\begin{aligned}
\left(\left(u_{1} \cdot \nabla\right) u_{1}-\left(u_{2} \cdot \nabla\right) u_{2}, u_{12}\right) & =\left(\left(u_{1} \cdot \nabla\right) u_{12}, u_{12}\right) \\
+\left(\left(u_{12} \cdot \nabla\right) u_{2}, u_{12}\right) & =\left(\left(u_{12} \cdot \nabla\right) u_{2}, u_{12}\right)
\end{aligned}
$$

and

$$
\begin{aligned}
\left(u_{1} \cdot \nabla \theta_{1}-u_{2} \cdot \nabla \theta_{2}, \theta_{12}\right) & =\left(u_{1} \cdot \nabla \theta_{12}, \theta_{12}\right) \\
+\left(u_{12} \cdot \nabla \theta_{2}, \theta_{12}\right) & =\left(u_{12} \cdot \nabla \theta_{2}, \theta_{12}\right)
\end{aligned}
$$


(which hold thanks to $\operatorname{div} u_{1}=0$ and $\left.u_{1}\right|_{\Gamma}=0$ ) and also

$$
\begin{aligned}
& \left(\left|\nabla u_{1}\right|^{2}-\left|\nabla u_{2}\right|^{2}, \theta_{12}\right)=\left(\nabla\left(u_{1}+u_{2}\right),\left(\nabla u_{12}\right) \theta_{12}\right) \quad \text { and } \\
& \left(\theta_{1} g \cdot u_{1}-\theta_{2} g \cdot u_{2}, \theta_{12}\right)=\left(\theta_{1} g \cdot u_{12}, \theta_{12}\right)+\left(g \cdot u_{2}, \theta_{12}^{2}\right),
\end{aligned}
$$

we get, by testing $(3)$ by $(v, \tilde{v}):=\left(u_{12}, \theta_{12}\right)$,

$$
\begin{aligned}
\nu \| \nabla & u_{12}\left\|_{2}^{2}+\kappa\right\| \nabla \theta_{12} \|_{2}^{2}+\left(b \theta_{12}, \theta_{12}\right)_{\Gamma} \\
= & \left(h_{12}, \theta_{12}\right)_{\Gamma}-\left(\left(u_{12} \cdot \nabla\right) u_{2}, u_{12}\right)-\left(u_{12} \cdot \nabla \theta_{2}, \theta_{12}\right) \\
& -\alpha_{0}\left(\theta_{12}, g \cdot u_{12}\right)+\alpha_{1}\left(\nabla\left(u_{1}+u_{2}\right),\left(\nabla u_{12}\right) \theta_{12}\right) \\
& +\alpha_{2}\left(\theta_{1} g \cdot u_{12}, \theta_{12}\right)+\alpha_{2}\left(g \cdot u_{2}, \theta_{12}^{2}\right) \\
\leq & N_{\Gamma}\left\|h_{12}\right\|_{2}\left\|\theta_{12}\right\|_{W^{1,2}(\Omega)}+\left\|\nabla u_{2}\right\|_{2}\left\|u_{12}\right\|_{4}^{2}+\left\|u_{12}\right\|\left\|_{4}\right\| \nabla \theta_{2}\left\|_{2}\right\| \theta_{12} \|_{4} \\
& +\alpha_{0}\left\|\theta_{12}\right\|_{2}\|g\|_{\infty}\left\|u_{12}\right\|_{2}+\alpha_{1} N_{6,4}^{2}\left\|\nabla\left(u_{1}+u_{2}\right)\right\|_{6}\left\|\nabla u_{12}\right\|_{2}\left\|\theta_{12}\right\|_{6} \\
& +\alpha_{2}\left\|\theta_{1}\right\|_{6}\|g\|_{\infty}\left\|u_{12}\right\|_{6}\left\|\theta_{12}\right\|_{6}+\alpha_{2} N_{6,3}^{2}\|g\|_{\infty}\left\|u_{2}\right\|_{\infty}\left\|\theta_{12}\right\|_{2}^{2} .
\end{aligned}
$$

Using Young and Poincaré inequalities, all right-hand-side terms but $\left\|h_{12}\right\|_{2}^{2}$ can be absorbed in the left-hand side as (6) is assumed.

Remark 2 The above uniqueness proof suggests a modification of the existence proof by showing a Lipschitz continuity of the mapping $\vartheta \mapsto \theta$ and then to use Banach's fixed-point theorem instead of the Schauder one. The requirement of contractivity of $\vartheta \mapsto \theta$ would, however, require (6) quantitatively stronger.

Remark 3 Usually, we are given by $\nu, \kappa$, and $b_{\min }$. Then the choice of a suitable $\varrho$ needs further analysis and (6) needs, in particular, a very small Reynolds number, cf. [2] for quantitative estimates.

\section{The optimal control problem}

We will consider the velocity/temperature tracking problem. For the purpose of Proposition 10 below, we consider two different norms for velocity, distinguished by $\xi=0,1$, and then the optimal-control problem:

(’)

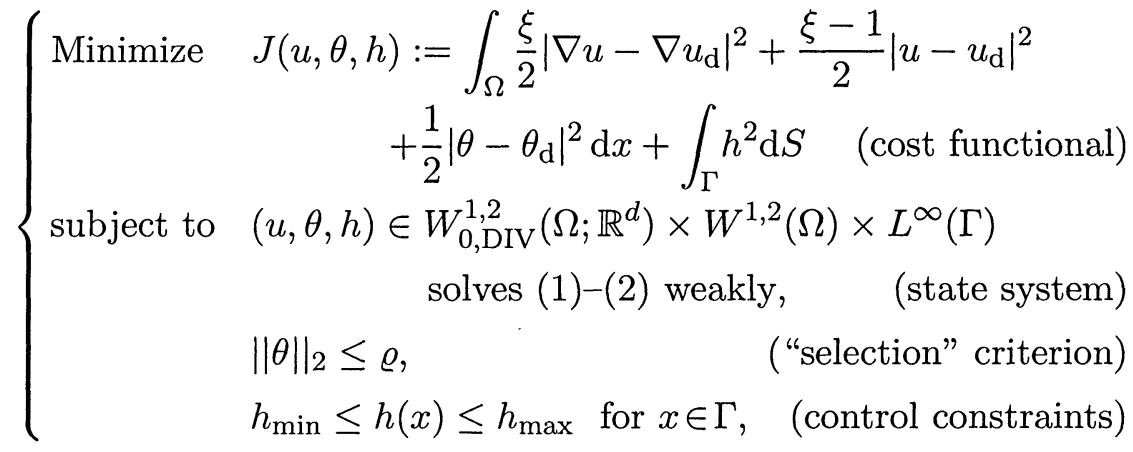


where $u_{\mathrm{d}}$ and $\theta_{\mathrm{d}}$ are the desired velocity and temperature profiles. Let us emphasize that $(\mathfrak{P})$ is not a linear/quadratic optimization problem because the controlled system (1), containing 4 bilinear/quadratic terms, is obviously nonlinear.

Remark 4 (The selection criterion.) The criterion $\|\theta\|_{2} \leq \varrho$ uses $\varrho$ from Proposition 1 and is not a state constraint in the usual sense because it does not constrain implicitly the controls but it only wants to avoid a possible nonuniqueness in the response. This selection criterion can be omitted for the Oberbeck-Boussinesq system (i.e. if $\alpha_{1}=\alpha_{2}=0$ ).

Proposition 5 Let $u_{\mathrm{d}}$ belong to $L^{2}\left(\Omega ; \mathbb{R}^{d}\right)($ if $\xi=0)$ or to $W^{1,2}\left(\Omega ; \mathbb{R}^{d}\right)$ (if $\xi=1), \theta_{\mathrm{d}} \in L^{2}(\Omega)$, and the assumptions (5)-(6) be satisfied. Then $(\mathfrak{P})$ has a solution.

Proof. The set $\mathfrak{H}_{\text {ad }}$ of admissible controls is compact in weak $L^{2}$ topology in which the state mapping $h \mapsto(u(h), \theta(h)),\|\theta\|_{2} \leq \varrho$, is continuous and the cost functional $J$ lower-semicontinuous. Hence the minimum of $\Phi(h)=J(u(h), \theta(h), h)$ does exist by Bolzano-Weierstraß' theorem.

\section{Optimality conditions}

Of course, since the controlled system is nonlinear, there may exists (beside the globally optimal control whose existence has been claimed in Proposition 5) also locally optimal controls. Let us consider a fixed locally optimal reference pair $(\bar{u}, \bar{\theta}, \bar{h})$. We begin with the first-order optimality conditions. Formally, they can be found by applying the wellknown Lagrange principle, where the state-equations are eliminated by the Lagrange function

$$
\begin{aligned}
L(u, \theta, h, w, \vartheta) & =J(u, \theta, h)-((u \cdot \nabla) u, w)-\nu(\nabla u, \nabla w) \\
& -\kappa(\nabla \theta, \nabla \vartheta)-(u \cdot \nabla \theta, \vartheta)+(h-b \theta, \vartheta)_{\Gamma} \\
& +\left(\left(1-\alpha_{0} \theta\right) g, w\right)+\left(\alpha_{1}|\nabla u|^{2}+\alpha_{2} \theta g \cdot u, \vartheta\right),
\end{aligned}
$$

cf. (3). Obviously, for fixed multipliers $w \in W_{0, \mathrm{DIV}}^{1,2}\left(\Omega ; \mathbb{R}^{d}\right)$ and $\vartheta \in$ $W^{1,2}(\Omega)$, the function $L(\cdot, \cdot, \cdot, w, \vartheta): W_{0, \mathrm{DIV}}^{1,2}\left(\Omega ; \mathbb{R}^{d}\right) \times W^{1,2}(\Omega) \times L^{2}(\Gamma) \rightarrow$ $\mathbb{R}$ is quadratic and continuous, hence it is a $C^{2}$-function, too. According to the Lagrange principle, $(\bar{u}, \bar{\theta}, \bar{h})$ should satisfy the necessary optimality conditions for minimizers of $L$ with respect to $h \in \mathfrak{H}_{\text {ad }}$, i.e.

$$
\begin{array}{ll}
{\left[L_{u}^{\prime}(\bar{u}, \bar{\theta}, \bar{h}, w, \vartheta)\right](u)=0} & \forall u \in W_{0, \mathrm{DIV}}^{1,2}\left(\Omega ; \mathbb{R}^{d}\right), \\
{\left[L_{\theta}^{\prime}(\bar{u}, \bar{\theta}, \bar{h}, w, \vartheta)\right](\theta)=0} & \forall \theta \in W^{1,2}(\Omega), \\
{\left[L_{h}^{\prime}(\bar{u}, \bar{\theta}, \bar{h}, w, \vartheta)\right](h-\bar{h}) \geq 0} & \forall h \in \mathfrak{H}_{\mathrm{ad}} .
\end{array}
$$


The identities $(18 \mathrm{a}, \mathrm{b})$ lead to the adjoint system which has (in the classical formulation) the form:

$$
\begin{aligned}
(\nabla \bar{u})^{\top} w-(\bar{u} \cdot \nabla) w-\nu \Delta w+\nabla \pi & =2 \alpha_{1} \operatorname{div}(\vartheta \nabla \bar{u})+\alpha_{2} \bar{\theta} g \vartheta \\
& +\bar{\theta} \nabla \vartheta+ \begin{cases}\bar{u}-u_{\mathrm{d}} & \text { for } \xi=0 \\
\Delta\left(u_{\mathrm{d}}-\bar{u}\right) & \text { for } \xi=1\end{cases} \\
\operatorname{div} w & =0 \\
\kappa \Delta \vartheta+\bar{u} \nabla \vartheta+\alpha_{2} g \cdot \bar{u} \vartheta & =\alpha_{0} g \cdot w+\theta_{\mathrm{d}}-\bar{\theta}
\end{aligned}
$$

with the boundary conditions

$$
w=0, \quad \kappa \frac{\partial \vartheta}{\partial n}+b \vartheta=0 \quad \text { on } \Gamma,
$$

for the so-called adjoint velocity, pressure, and temperature $(w, \pi, \vartheta) \in$ $W_{0, \mathrm{DIV}}^{1,2}\left(\Omega ; \mathbb{R}^{d}\right) \times L^{2}(\Omega) \times W^{1,2}(\Omega)$ associated with a given state $(\bar{u}, \bar{\theta})$. Note that $(\nabla u)^{\top} w-(u \cdot \nabla) w$ means $\left(\sum_{k=1}^{n}\left(\frac{\partial u_{k}}{\partial x_{i}} w_{k}-u_{k} \frac{\partial w_{i}}{\partial x_{k}}\right)\right)_{\xi=1, \ldots, n}$.

Proposition 6 (1st-order necessary conditions.) Let (5)-(6) hold, and let $\bar{h}$ be a locally optimal control for $(\mathfrak{P})$ with associated state $(\bar{u}, \bar{\theta})$. Then the variational inequality

$$
\bar{h}(x)= \begin{cases}h_{\max } & \text { if }-\vartheta(x)>2 h_{\max }, \\ -\vartheta(x) / 2 & \text { if }-\vartheta(x) \in\left[2 h_{\min }, 2 h_{\max }\right], \\ h_{\min } & \text { if }-\vartheta(x)<2 h_{\min }\end{cases}
$$

is satisfied for $\vartheta=\vartheta(\bar{u}, \bar{\theta}) \in W^{1,2}(\Omega)$ being, together with $w=w(\bar{u}, \bar{\theta})$, the unique weak solution to the adjoint system (19)-(20).

Proof. (Sketched.) In view of the special form (4) of $\mathfrak{H}_{\mathrm{ad}},(18 \mathrm{c})$ is equivalent to (21) and, as already told, $(18 \mathrm{a}, \mathrm{b})$ is equivalent to the adjoint system (19)-(20) whose solution can be shown to exist under the assumption (5)-(6).

To perform 2nd-order analysis (as, in the context of fluid control used in $[10,18,27])$, we need 2 nd-order derivative of the Lagrange function. In view of $(17)$, the 2 nd differential of $L(\cdot, \cdot, \cdot, w, \vartheta)$ at a point $(u, \theta, h)$ is

$$
\begin{aligned}
L_{(u, \theta, h)}^{\prime \prime}( & u, \theta, h, w, \vartheta)\left[\left(u_{1}, \theta_{1}, h_{1}\right),\left(u_{2}, \theta_{2}, h_{2}\right)\right]=-\left(\left(u_{1} \cdot \nabla\right) u_{2}, w\right) \\
& -\left(\left(u_{2} \cdot \nabla\right) u_{1}, w\right)-\left(u_{1} \cdot \nabla \theta_{2}, \vartheta\right)-\left(u_{2} \cdot \nabla \theta_{1}, \vartheta\right) \\
& +2 \alpha_{1}\left(\vartheta \nabla u_{1}, \nabla u_{2}\right)+\alpha_{2}\left(\theta_{1} g \cdot u_{2}, \vartheta\right)+\alpha_{2}\left(\theta_{2} g \cdot u_{1}, \vartheta\right) \\
& +\left(\theta_{1}, \theta_{2}\right)+\left(h_{1}, h_{2}\right)_{\Gamma}+ \begin{cases}\left(u_{1}, u_{2}\right) & \text { if } \xi=0 \\
\left(\nabla u_{1}, \nabla u_{2}\right) & \text { if } \xi=1 .\end{cases}
\end{aligned}
$$


This quadratic form is therefore independent of $(u, \theta, f)$ and bounded provided the multipliers $w$ and $\vartheta$ are bounded in $L^{\infty}$-norm, as indeed shown in the proof of Proposition 10 below under the assumption $b$ smooth and, for $\gamma=2$,

$$
\theta_{\mathrm{d}} \in L^{\gamma}(\Omega), \quad u_{\mathrm{d}} \in \begin{cases}L^{\gamma}\left(\Omega ; \mathbb{R}^{d}\right) & \text { if } \xi=0 \\ W^{2, \gamma}\left(\Omega ; \mathbb{R}^{d}\right) & \text { if } \xi=1\end{cases}
$$

By using Green formula and $\operatorname{div} \tilde{u}=0$ and $\left.\tilde{u}\right|_{\Gamma}=0,(22)$ restricted on the diagonal $\left(u_{1}, \theta_{1}, h_{1}\right)=\left(u_{2}, \theta_{2}, h_{2}\right)$ becomes symmetric and takes the form

$$
\begin{aligned}
& L_{(u, \theta, h)}^{\prime \prime}(u, \theta, h, w, \vartheta)(\tilde{u}, \tilde{\theta}, \tilde{h})^{2}=2((\tilde{u} \cdot \nabla) w, \tilde{u})+2(\tilde{u} \cdot \nabla \vartheta, \tilde{\theta}) \\
& +2 \alpha_{1}(\vartheta \nabla \tilde{u}, \nabla \tilde{u})+2 \alpha_{2}(\tilde{\theta} g, \tilde{u} \vartheta)+\|\tilde{h}\|_{2, \Gamma}^{2}+\|\tilde{\theta}\|_{2}^{2}+ \begin{cases}\|\tilde{u}\|_{2}^{2} & \text { if } \xi=0 \\
\|\nabla \tilde{u}\|_{2}^{2} & \text { if } \xi=1 .\end{cases}
\end{aligned}
$$

Proposition 7 (2nd-order sufficient conditions.) Let (5)-(6) be valid, $b$ smooth, (23) hold with $\gamma=2$, and let $(\bar{u}, \bar{\theta}, \bar{h}, w, \vartheta)$ satisfy (1)-(2), $\bar{h} \in \mathfrak{H}_{\mathrm{ad}}$, the first-order necessary conditions (19)-(20), together with the second-order sufficient condition:

$$
L_{(u, \theta, h)}^{\prime \prime}(\bar{u}, \bar{\theta}, \bar{h}, w, \vartheta)(u, \theta, h)^{2} \geq \delta\|h\|_{2, \Gamma}^{2}
$$

for $L_{(u, \theta, h)}^{\prime \prime}$ from $(24)$, some $\delta>0$, and for all $(u, \theta, h)$ solving the system (1)-(2) linearized at $(\bar{u}, \bar{\theta}, \bar{h})$, i.e. for $(u, \theta, h)$ satisfying $(1 \mathrm{~b}),(2)$, and

$$
\begin{aligned}
& (u \cdot \nabla) \bar{u}+(\bar{u} \cdot \nabla) u-\nu \Delta u+\nabla p=-g \alpha_{0} \theta, \\
& u \cdot \nabla \bar{\theta}+\bar{u} \cdot \nabla \theta-\kappa \Delta \theta=-2 \alpha_{1}(\nabla \bar{u}, \nabla u)+\alpha_{2} g \cdot(\theta \bar{u}+\bar{\theta} u)
\end{aligned}
$$

in the weak sense. Then $(\bar{u}, \bar{\theta}, \bar{h})$ is locally optimal with respect to the topology of $W^{1,2}\left(\Omega ; \mathbb{R}^{d+1}\right) \times L^{2}(\Gamma)$.

Proof. (Sketched.) By [6], (25)-(26) yields $\Phi^{\prime \prime}(\bar{h})(h, h) \geq \delta_{1}\|h\|_{2, \Gamma}^{2}$ for some $\delta_{1}>0$ and for all $h \in \mathfrak{H}_{\mathrm{ad}}$. Moreover, one can prove that $(u, \theta, h, w, \vartheta) \mapsto L_{(u, \theta, h)}^{\prime \prime}(u, \theta, h, w, \vartheta)$ is continuous, and this continuity is inherited also by $\Phi^{\prime \prime}(\cdot)(h, h)$, so that one can conclude that $\bar{h}$ is locally optimal for $\Phi$ with respect to the norm of $L^{2}(\Gamma)$. By the continuity of the state mapping $h \mapsto(u, \theta): L^{2}(\Gamma) \rightarrow W^{1,2}\left(\Omega ; \mathbb{R}^{d+1}\right)$, see Proposition 1 , this gives the claimed local optimality of $(\bar{u}, \bar{\theta}, \bar{h})$.

\section{The increment formula}

For $h \in \mathfrak{H}_{\mathrm{ad}}$, as in the proof of Proposition 5 , we denote $\Phi(h)=$ $J(u(h), \theta(h), h)$ with $\|\theta(h)\|_{2} \leq \varrho$. Recall that $\operatorname{such} \theta=\theta(h)$ and $u=$ $u(h)$ are unique under the assumption (5)-(6). 
For the following lemma, let us abbreviate $z:=(u, \theta) \in Z:=$ $W_{0, \mathrm{DIV}}^{1,2}\left(\Omega ; \mathbb{R}^{d}\right) \times W^{1,2}(\Omega)$ and $H=L^{2}(\Gamma)$, and define $\Pi: Z \times H \rightarrow Z^{*}$ by $\langle\Pi(z, h), \tilde{z}\rangle=$ the left-hand side of $(3)$; thus $\Pi(z, h)=0$ is just (3).

Lemma 8 (Increment formula for bi-quadratic problems.) Let $J: Z \times$ $H \rightarrow \mathbb{R}$ and $\Pi: Z \times H \rightarrow Z^{*}$ be quadratic mappings and $\lambda \in Z^{* *} \cong Z$ satisfy $L_{z}^{\prime}(z, h, \lambda)=0$ with $L(z, h, \lambda)=J(z, h)-\lambda \circ \Pi(z, h)$, and let $\Pi(z, h)=0$ and $\Pi(\tilde{z}, \tilde{h})=0$. Then

$$
\Phi(\tilde{h})-\Phi(h)-\Phi^{\prime}(h)(\tilde{h}-h)=\frac{1}{2} L_{(z, h)}^{\prime \prime}(z, h, \lambda)(\tilde{z}-z, \tilde{h}-h)^{2} .
$$

Proof. As $L(\cdot, \cdot, \lambda)$ is quadratic, the Taylor expansion up to 2nd-order term is exact, i.e.

$$
\begin{aligned}
L(\tilde{z}, \tilde{h}, \lambda)=L(z, h, \lambda) & +L_{(z, h)}^{\prime}(z, h, \lambda)(\tilde{z}-z, \tilde{h}-h) \\
& +\frac{1}{2} L_{(z, h)}^{\prime \prime}(z, h, \lambda)(\tilde{z}-z, \tilde{h}-h)^{2} .
\end{aligned}
$$

As $\Pi(z, h)=0, L(z, h, \lambda)=J(z, h)-\lambda \circ \Pi(z, h)=\Phi(h)$. Similarly, $\Pi(\tilde{z}, \tilde{h})=0$ implies $L(\tilde{z}, \tilde{h}, \lambda)=\Phi(\tilde{h})$. Moreover, using the adjoint equation $L_{z}^{\prime}(z, h, \lambda)=0$, we get

$$
\begin{array}{r}
L_{(z, h)}^{\prime}(z, h, \lambda)(\tilde{z}-z, \tilde{h}-h)=L_{z}^{\prime}(z, h, \lambda)(\tilde{z}-z)+L_{h}^{\prime}(z, h, \lambda)(\tilde{h}-h) \\
=J_{h}^{\prime}(z, h)(\tilde{h}-h)-\left[\Pi_{h}^{\prime}(z, h)(\tilde{h}-h)\right]^{*} \lambda=\Phi^{\prime}(h)(\tilde{h}-h) .
\end{array}
$$

As the controlled system (1) as well as the cost functional in (P) are quadratic, we can use Lemma 8 for our problem:

Corollary 9 The following increment formula for (P) holds:

$$
\begin{aligned}
& J(\tilde{u}, \tilde{\theta}, \tilde{h})-J(u, \theta, h)-(\vartheta+2 h, \tilde{h}-h)_{\Gamma} \\
& \quad=(((\tilde{u}-u) \cdot \nabla) w, \tilde{u}-u)+((\tilde{u}-u) \cdot \nabla \vartheta, \tilde{\theta}-\theta) \\
& \quad+\alpha_{1}\left(\vartheta(\nabla(\tilde{u}-u), \nabla(\tilde{u}-u))+\alpha_{2}((\tilde{\theta}-\theta) g,(\tilde{u}-u) \vartheta)\right. \\
& \quad+\frac{1}{2}\|\tilde{h}-h\|_{2, \Gamma}^{2}+\frac{1}{2}\|\tilde{\theta}-\theta\|_{2}^{2}+ \begin{cases}\frac{1}{2}\|\tilde{u}-u\|_{2}^{2} & \text { if } \xi=0 \\
\frac{1}{2}\|\nabla(\tilde{u}-u)\|_{2}^{2} & \text { if } \xi=1 .\end{cases}
\end{aligned}
$$

where $\tilde{u}=u(\tilde{f}), u=u(f), w=w(u, \theta)$, and $\vartheta=\vartheta(u, \theta)$.

Proof. Use (27) with $L^{\prime \prime}$ from $(24)$ and $\lambda=(w, \vartheta)$, and realize that $\Phi^{\prime}(h)=L_{h}^{\prime}(u, \theta, h, w, \vartheta)=\vartheta+2 h$.

The formula (30) can be used for global analysis of $(\mathfrak{P})$ provided one shows the multipliers $w$ and $\vartheta$ sufficiently small in the $W^{1, \infty}$-norm. 
Proposition 10 Let (5)-(6) be valid, b smooth, (23) hold for $\gamma>d$, let $\xi=1$ or $\alpha_{1}=0$, and, for any $h \in \mathfrak{H}_{\mathrm{ad}}$, the corresponding adjoint variables $(w, \vartheta)$ satisfy

$$
\begin{aligned}
& \frac{1}{2} \geq A_{w, \vartheta}:=\left\{\begin{aligned}
&\|\nabla w\|_{\infty}+\frac{1}{2}\|\nabla \vartheta\|_{\infty}+\frac{1}{2} \alpha_{2}\|g\|_{\infty}\|\vartheta\|_{\infty} \text { if } \xi=0, \\
& N_{2}^{2}\left(\|\nabla w\|_{\infty}+\frac{1}{2}\|\nabla \vartheta\|_{\infty}\right. \\
&\left.+\frac{1}{2} \alpha_{2}\|g\|_{\infty}\|\vartheta\|_{\infty}\right)+\alpha_{1}\|\vartheta\|_{\infty} \text { if } \xi=1,
\end{aligned}\right. \\
& \frac{1}{2} \geq A_{\vartheta}:=\frac{1}{2}\|\nabla \vartheta\|_{\infty}+\frac{1}{2} \alpha_{2}\|g\|_{\infty}\|\vartheta\|_{\infty} .
\end{aligned}
$$

Then $\Phi$ is strictly convex on $\mathfrak{H}_{\text {ad }}$.

Proof. First, let us prove $W^{1, \infty}$-estimates of the multipliers $w$ and $\vartheta$. The adjoint equation (19) can equally be viewed as the Stokes system and the Poisson equation with the right-hand sides

$$
\begin{aligned}
f_{1}= & -(\bar{u} \cdot \nabla) \bar{w}+(\nabla \bar{u})^{\top} \bar{w}+2 \alpha_{1} \nabla \vartheta \cdot \nabla \bar{u}+2 \alpha_{1} \vartheta \Delta \bar{u} \\
& +\alpha_{2} \bar{\theta} g \cdot \vartheta-\bar{\theta} \nabla \vartheta+ \begin{cases}\bar{u}-u_{\mathrm{d}} & \text { for } \xi=0 \\
\Delta\left(u_{\mathrm{d}}-\bar{u}\right) & \text { for } \xi=1\end{cases} \\
f_{2}= & \bar{u} \nabla \vartheta+\alpha_{2} g \cdot \bar{u} \vartheta+\bar{\theta}-\theta_{\mathrm{d}} .
\end{aligned}
$$

By the proof of Proposition 1 , we know that $\bar{u} \in W^{2,2}\left(\Omega ; \mathbb{R}^{d}\right)$ and $\bar{\theta} \in$ $W^{1,2}(\Omega)$, so that $f:=(\bar{u} \cdot \nabla) \bar{u}+\alpha_{0} g \theta \in L^{6}\left(\Omega ; \mathbb{R}^{d}\right)$ and, by the $W^{2, p_{-}}$ regularity [14] of Stokes' system with the right-hand side $f$, we get $\bar{u} \in$ $W^{2, \gamma}\left(\Omega ; \mathbb{R}^{d}\right) ;$ as $n \leq 3$ we may assume $\gamma \leq 6$. Besides, certainly $w \in$ $W_{0, \mathrm{DIV}}^{1,2}\left(\Omega ; \mathbb{R}^{d}\right)$ and $\vartheta \in W^{1,2}(\Omega)$. As $W^{1,2}(\Omega) \subset L^{6}(\Omega)$ and $W^{2, \gamma}(\Omega) \subset$ $W^{1, \infty}(\Omega)$, we can see that $f_{2} \in L^{2}(\Omega)$ due to the estimate

$$
\left\|f_{2}\right\|_{2} \leq\|\bar{u}\|_{\infty}\|\nabla \vartheta\|_{2}+\alpha_{2} N_{6,2}\|g\|_{\infty}\|\bar{u}\|_{\infty}\|\vartheta\|_{6}+\left\|\bar{\theta}-\theta_{\mathrm{d}}\right\|_{2} .
$$

By the $W^{2,2}$-regularity of the equation $\kappa \Delta \vartheta+f_{2}=0$ with the boundary conditions $\kappa \partial \vartheta / \partial n+b \vartheta=0$ (here we need $b$ smooth), we can see that $\vartheta \in W^{2,2}(\Omega)$. Then we have also $f_{1} \in L^{2}\left(\Omega ; \mathbb{R}^{d}\right)$ due to the estimate

$$
\begin{aligned}
\left\|f_{1}\right\|_{2} \leq & \|\bar{u}\|_{\infty}\|\nabla \bar{w}\|_{2}+N_{6,4}^{2}\|\nabla \bar{u}\|_{6}\|\bar{w}\|_{6}+2 \alpha_{1} N_{6,4}^{2}\|\nabla \vartheta\|_{6}\|\nabla \bar{u}\|_{6} \\
& +2 \alpha_{1}\|\vartheta\|_{\infty}\|\Delta \bar{u}\|_{2}+\alpha_{2} N_{\infty, 2}\|\bar{\theta}\|_{\infty}\|g\|_{\infty}\|\vartheta\|_{\infty} \\
& +N_{6,2}\|\bar{\theta}\|_{\infty}\|\nabla \vartheta\|_{6}+ \begin{cases}N_{\infty, 2}\left\|\bar{u}-u_{\mathrm{d}}\right\|_{\infty} & \text { for } \xi=0 \\
\left\|\Delta\left(u_{\mathrm{d}}-\bar{u}\right)\right\|_{2} & \text { for } \xi=1\end{cases}
\end{aligned}
$$

Again by regularity for Stokes' systems [14; Chap. IV, Thm.6.1], we have $w \in W^{2,2}\left(\Omega ; \mathbb{R}^{d}\right)$. By usual bootstrap argument, we can see that $f_{2}$ then belongs even to $L^{\gamma}(\Omega)$ (recall that $\gamma \leq 6$ ) due to the estimate

$$
\begin{aligned}
\left\|f_{2}\right\|_{\gamma} \leq & N_{6, \gamma}\|\bar{u}\|_{\infty}\|\nabla \vartheta\|_{6} \\
& +\alpha_{2} N_{\infty, \gamma}\|g\|_{\infty}\|\bar{u}\|_{\infty}\|\vartheta\|_{\infty}+\left\|\bar{\theta}-\theta_{\mathrm{d}}\right\|_{\gamma} .
\end{aligned}
$$


Hence, we get one of the desired estimates: $\vartheta \in W^{2, \gamma}(\Omega) \subset W^{1, \infty}(\Omega)$. Then, by the obvious "bootstrap" modification of (35), we have also $f_{1} \in L^{\gamma}\left(\Omega ; \mathbb{R}^{d}\right)$. Again by $W^{2, \gamma}$-regularity for Stokes' system [14], we have the remaining desired estimate $w \in W^{2, \gamma}\left(\Omega ; \mathbb{R}^{d}\right) \subset W^{1, \infty}\left(\Omega ; \mathbb{R}^{d}\right)$.

Then, in the case $\xi=1$, we can estimate the terms with indefinite signs in (30) by the Hölder and Young inequalities as follows:

$$
\begin{aligned}
& (((\tilde{u}-u) \cdot \nabla) w, \tilde{u}-u)+((\tilde{u}-u) \cdot \nabla \vartheta, \tilde{\theta}-\theta) \\
& \quad+\alpha_{1}(\vartheta \nabla(\tilde{u}-u), \nabla(\tilde{u}-u))+\alpha_{2}((\tilde{\theta}-\theta) g,(\tilde{u}-u) \vartheta) \\
& \quad \geq-\|\tilde{u}-u\|_{2}^{2}\|\nabla w\|_{\infty}-\|\tilde{u}-u\|_{2}\|\nabla \vartheta\|_{\infty}\|\tilde{\theta}-\theta\|_{2} \\
& \quad-\alpha_{1}\|\vartheta\|_{\infty}\|\nabla(\tilde{u}-u)\|_{2}^{2}-\alpha_{2}\|\tilde{u}-u\|_{2}\|g\|_{\infty}\|\vartheta\|_{\infty}\|\tilde{\theta}-\theta\|_{2} \\
& \quad \geq-A_{w, \vartheta}\|\nabla(\tilde{u}-u)\|_{2}^{2}-A_{\vartheta}\|\tilde{\theta}-\theta\|_{2}^{2} .
\end{aligned}
$$

Then (31) ensures that these terms are dominated by last two terms in (30) so that $\Phi(\tilde{h})-\Phi(h)-\Phi^{\prime}(h)(\tilde{h}-h) \geq 0$ by (27). Replacing the role of $h$ and $\tilde{h}$, we get $\Phi(h)-\Phi(\tilde{h})-\Phi^{\prime}(\tilde{h})(h-\tilde{h}) \geq 0$, and by adding these inequalities we get monotonicity of $\Phi^{\prime}$, hence convexity of $\Phi$. The term $\frac{1}{2}\|\tilde{h}-h\|_{2, \Gamma}^{2}$ in (30) then ensures strict convexity of $\Phi$.

For $\alpha_{1}=0$, we can handle the case $\xi=0$ by simplified estimation as suggested in (31a).

Corollary 11 (1st-order sufficient condition.) Under the conditions in Proposition 10, (21) ensures that $\bar{h}$ is the unique optimal control to $(\mathfrak{P})$.

Remark 12 The increment-formula technique has been developed in a general context in [13]. Increment formula (30) has been derived in [23] for mere Navier-Stokes system (in particular, the $W^{1, \infty}$-regularity of $w$ has been proved by Málek [23] for this case) and in [2] for OberbeckBoussinesq system (i.e. $\alpha_{1}=\alpha_{2}=0$ ). Severe restrictions of conditions like (31) have been analyzed by Bubák [2].

\section{References}

[1] N. Bilić: Approximation of optimal distributed control problem for Navier-Stokes equations. In: Numerical Methods and Approx. Th., Univ. Novi Sad, Novi Sad, $1985,177-185$.

[2] P. Bubák: Optimal control of flow driven by the thermal field. MS-diploma thesis, Math.-Phys. Faculty, Charles University, Prague, 2002.

[3] J. Burkard, J.Peterson: Control of steady incompressible 2D channel flow. In: Flow Control (M.D.Gunzburger, ed.), IMA Vol. Math. Appl. 68, Springer, New York, 1995, 111-126.

[4] A. Capatina, R. Stavre: Optimal control of a non isothermal Navier-Stokes flow. Int. J. Eng. Sci. 34 (1996), 59-66.

[5] E. Casas: Optimality conditions for some control problems of turbulent flow. In: Flow Control (M.D.Gunzburger, ed.), IMA Vol. Math. Appl. 68, Springer, New York, 1995, 127-147. 
[6] E. Casas, F. Tröltzsch: Second order necessary and sufficient optimality conditions for optimization problems and applications to control theory, to appear in SIAM J. Optimization.

[7] P.Constantin, P.Foias: Navier-Stokes equations. The University of Chicago Press, 1989.

[8] C. Cuvelier: Optimal control of a system governed by the Navier-Stokes equations coupled with the heat equation. In: New Dev. Differ. Equat. (W.Eckhaus, ed.) North-Holland, 1976, 81-98.

[9] C. Cuvelier: Resolution numerique d'un probleme de controle optimal d'un couplage des equations de Navier-Stokes et celle de la chaleur. Calcolo 15 (1980), 345-379.

[10] M.C. Desai, K. Ito: Optimal control of Navier-Stokes equations. SIAM J. Control Optim. 32 (1994), 1428-1446.

[11] H.O. Fattorini: Infinite Dimensional Optimization and Control Theory. Cambridge Univ. Press, Cambridge, 1999.

[12] A.V.Fursikov: Optimal Control of Distributed Systems. Theory and Applications. AMS, Providence, 2000.

[13] R. Gabasov, F. Kirillova: The Qualitative Theory of Optimal Processes. Marcel Dekker, New York, 1976 (Russian orig.: Nauka, Moscow, 1971).

[14] P.G. Galdi: An introduction to the Navier-Stokes Equations. Springer-Verlag, 1994.

[15] M.D. Gunzburger: A prehistory of flow control and optimization. In: Flow Control (M.D.Gunzburger, ed.), IMA Vol. Math. Appl. 68, Springer, New York, 1995, 185-195.

[16] M.D. Gunzburger, L.Hou, T.P.Svobodny: Analysis and finite element approximation of optimal control problems for stationary Navier-Stokes equations with distributed and Neumann controls. Math. Comp. 57 (1991), 123-151.

[17] M.D. Gunzburger, L. Hou, T.P. Svobodny: Boundary velocity control of incompressible flow with an application to viscous drag reduction. SIAM J. Control Optim. 30 (1992), 167-181.

[18] M. Hinze: A remark on second order methods in control of fluid flow. Zeitschift Angew. Math. Mech. 81, Suppl. 3, (2001).

[19] K.Ito, J.S.Scroggs, H.T. Tran: Optimal control of thermally coupled NavierStokes equations. In: Optimal design and control. (J.Borggaard et al., eds.) Birkhäuser, Boston, 1995, 199-214.

[20] K.Ito, H.T. Tran, J.S. Scroggs: Mathematical issues in optimal design of a vapor transport reactor. In: Flow Control (M.D.Gunzburger, ed.), IMA Vol. Math. Appl. 68, Springer, New York, 1995, 197-218.

[21] Y.Kagei, M. Růžička, G. Thäter: Natural Convection with Dissipative Heating. Comm. Math. Physics, 214 (2000), 287-313.

[22] J.L. Lions: Contrôle des systémes distribués singuliers. Bordas, Paris, 1983. Engl. transl.: Control of Distributed Singular Systems. Gauthier-Villars, 1985.

[23] J. Málek, T. Roubíček: Optimization of steady flows for incompressible viscous fluids. In: Nonlinear Applied Analysis. (Eds. A.Sequiera, H. Beirão da Vega, J.H.Videman) Plenum Press, New York, 1999, 355-372.

[24] B. Mohammadi, O.Pironneau: Analysis of the K-Epsilon turbulence model. J.Wiley, Chichester, 1994.

[25] K.R. Rajagopal, M.Růžička, A.R. Srinivasa: On the Oberbeck-Boussinesq Approximation. Math. Models Methods Appl. Sci. 6 (1996), 1157-1167.

[26] T.Roubíček: Steady-state buoyancy-driven viscous flow with measure data. Mathematica Bohemica, 126, (2001), 493-504.

[27] T. Roubíček, F. Tröltzsch: Lipschitz stability of optimal controls for the steadystate Navier-Stokes equations. Control \& Cybernetics, submitted. 